\title{
Data Mining mit Herz
}

A

1s Storyteller ist Datenerfassung und -analyse Teil meines Handwerkzeugs. Dramaturgische Muster wie die Heldenreise wären niemals entdeckt worden, wenn Joseph Campbell sich nicht die Mühe gemacht hätte, eine datenbasierte Feldforschung zu betreiben. Er hockte sich mit Ureinwohnern und Naturvölkern ans Lagerfeuer, um zu hören, welche Muster ihren Erzählungen zugrunde lagen. Und entdeckte dabei, dass tatsächlich jede Geschichte auf eine Urform zurückgeht.

Heute funktioniert Data Mining anders. Wir wenden statistische Methoden an, um neue Trends und Querverbindungen in großen Datenbeständen zu erkennen. Dazu nutzen wir computergestützte Methoden, um die Massen an Daten zu bewältigen.

Nichts anderes machen unser Körper und unser Geist schon seit Urzeiten. Wir produzieren und analysieren millisekündlich Daten in Nanogenauigkeit und leben dadurch hocheffizient. Das schützt uns und hilft uns zu überleben. Zum Beispiel wenn wir schnell über die Autobahn rauschen. Da erhalten wir nämlich viel mehr Daten, als wir benötigen. Die zum sicheren Fahren unnötigen Infos sortieren wir direkt aus. Was für eine gewaltige Leistung, die die KI in selbstfahrenden Autos jetzt auch anwendet, indem sie Datenstrukturen erkennt und Handlungen ab- und anleitet.

Data Mining hat früh angefangen. Seit der Entstehung einfacher Zählverfahren vor 50.000 Jahren hat sich die Mathematik entwickelt, die den menschlich-technischen Kulturfortschritt massiv vorangetrieben hat. Auch sie dient der Beschreibung und Bearbeitung von einfachen und komplexen Mustern. Auch sie arbeitet mit vorgefundenen und selbstgeschaffenen Strukturen.

Als Galileo Galilei vom Fernrohr hört, ist ihm klar: „Damit kann ich in neue Welten vorstoßen.“ Er beobachtet die Jupitermonde, findet eine Berechnungsformel für deren Erscheinen und Verschwinden und versetzt die ganze Welt inklusive Papst und Kaiser in Aufregung. Die Umkehrung des
Fernrohrs, das Mikroskop, dringt dann in den Mikrokosmos vor. Zwei Werkzeuge, die uns ganz neue Datenquellen und Formeln zugänglich machen.

In meiner Lebenszeit kommen wieder andere Werkzeuge hinzu: Computer, Halbleiter, Computersprachen und sogar Quantencomputer. Damit bilden Datenanalyse und -auswertung einen immer mächtigeren Innovationshebel. Aber wie beim fernöstlichen Ying und Yang-Prinzip wächst mit der Ratio auch der Gegenpol, die diffus-menschliche Seite.

Je mehr Informationen wir haben, umso mehr Hass und Überforderung entstehen. Während die Technikunternehmen voller Glauben auf Big Data, Data Mining und kontrollierte random studies setzen, machen sich auf der anderen Seite verheerende Zustände breit. Fake News, Wutbürger, Handelsdefizite, Klimakatastrophen, digitale Geld- und Bitcoinvermehrung, ein politischer Rechtsruck und die totale Überladung des Bürgers mit Informationen.

Wie wäre es, wenn wir das Genie von Mathematik und Data-Mining auf den gesamten Planeten anwenden, anstatt auf maximalen Profit? In der echten Welt ist schließlich immer noch alles beschränkt. Die Wasser- und Sauerstoffmengen sind genauso konstant wie die Bodenfläche. Wir müssten mit einer Deckelung rechnen lernen.

Mit welcher Gleichung können wir also eine konstruktive Parallelexistenz von Rationalem und Emotionalem erreichen? Wie können wir Gefühle in Formeleinschübe packen, damit unser Weltbild wieder realer und ganzheitlicher wird? Und wie menschlichere Algorithmen bauen, die die vitale Vielfalt von Erde und Persönlichkeiten stützen, anstatt' sie auszubeuten? Fragen, die wir mithilfe von Data Mining klären können. Und müssen.

Uwe Walter ist Storytelling- und Change-Experte für Medien- und Industrieunternehmen. Er berät so unterschiedliche Kunden wie YouTube-Stars, Start-ups, Blogger, Verlage, Radio- und Fernsehsender sowie Filmproduktionen. Seine Expertise: Wie generiere ich Reichweite durch zukunftssicheres Erzählen? 